\title{
Hadron spectroscopy in LHCb
}

\author{
Antimo Palano ${ }^{1,3, \star}$ \\ ${ }^{1}$ INFN and University of Bari, Via Orabona 4, 70125 Bari, Italy
}

\begin{abstract}
The LHCb experiment is designed to study the properties and decays of heavy flavored hadrons produced in pp collisions at the LHC. The data collected in the LHC Run I enables precision spectroscopy studies of beauty and charm hadrons. The latest results on spectroscopy of conventional and exotic hadrons are reviewed. In particular the discovery of the first charmonium pentaquark states in the $J / \psi p$ system, the possible existence of four-quark states decaying to $J / \psi \phi$ and the confirmation of resonant nature of the $Z_{c}(4430)^{-}$mesonic state are discussed. In the sector of charmed baryons, the observation of five new $\Omega_{c}$ states, the observation of the $\Xi_{c c}^{+}$and the study of charmed baryons decaying to $D^{0} p$ are presented.
\end{abstract}

\section{Introduction}

In the simple quark model, only two types of quark combinations are required to account for the existing hadrons, i.e. $q \bar{q}$ combinations form mesons, while baryons are made up of three quarks. However, in the quark model proposed by Gell-Mann and Zweig in 1960s [1] other SU(3) colorneutral combinations of quarks and gluons such as $g g$ glueballs, $q \bar{q} g$ hybrids, $q \bar{q} q \bar{q}$ tetraquarks, $q \bar{q} q q q$ pentaquarks etc. are allowed. The world's largest data sample of beauty and charm hadrons collected by $\mathrm{LHCb}$ during LHC Run I provides great opportunities for studying the production and properties of heavy hadrons. The present document describes some recent results from $\mathrm{LHCb}$ in exotic and standard hadron spectroscopy. For all the studies the LHCb data corresponding to $3 \mathrm{fb}^{-1}$ of integrated luminosity in 7 and $8 \mathrm{TeV}$ pp collisions has been used.

\section{The observation of pentaquarks in $\Lambda_{b}^{0} \rightarrow J / \psi p K^{-}$}

Evidences/observations of pentaquarks candidates were reported around 2005 by several experiments, always with low statistics and approximate statistical treatment of the data. For a summary and references see ref. [2]. High statistics searches gave negative results (see for example ref. [3]).

LHCb experiment studied the decay of $\Lambda_{b}^{0} \rightarrow J / \psi p K^{-}$[4]. A sample of 26,007 \pm 166 signal candidates, on $5.4 \%$ background, within $\pm 15 \mathrm{MeV}$ of the $J / \psi p K^{-}$mass peak has been selected using the full Run I dataset. An anomalous peaking structure in the $J / \psi p$ invariant mass spectrum might be observed on the Dalitz plot shown in Fig. 1.

Since the contribution of $\Lambda^{*} \rightarrow K^{-} p$ resonances corresponding to the vertical bands only dominates the low $m^{2}(K p)$ region, it is unlikely that the horizontal band is an effect of interference between

^e-mail: antimo.palano@ba.infn.it 


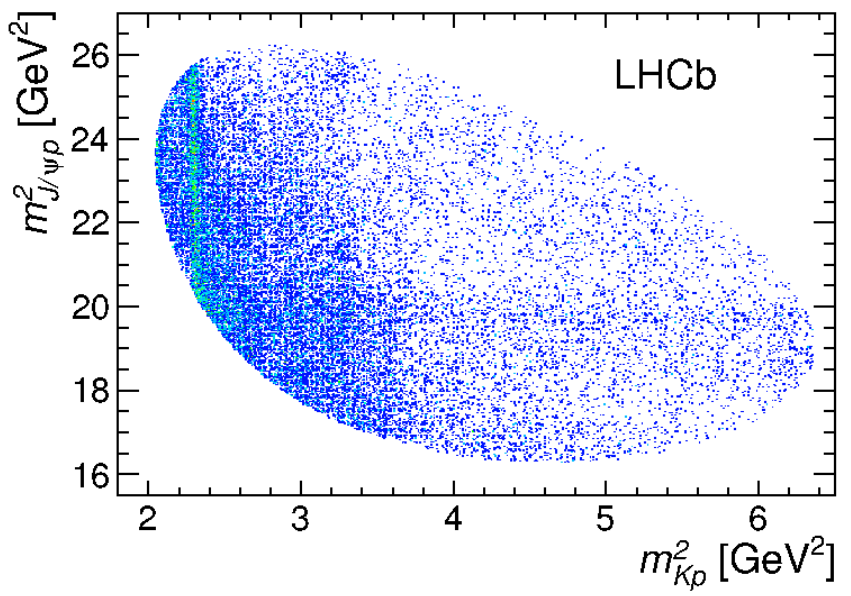

Figure 1. Dalitz plot of $\Lambda_{b}^{0} \rightarrow J / \psi p K^{-}$.

the $\Lambda^{*}$ states. This structure can also be seen in the invariant mass projections shown in Fig. 2 . If the peak structure observed in Fig. 2(b) represents a resonance strongly decaying into $J / \psi p$, the minimal valence quarks should be $c \bar{c} u u d$, a charmonium pentaquark state. A full six-dimensional amplitude
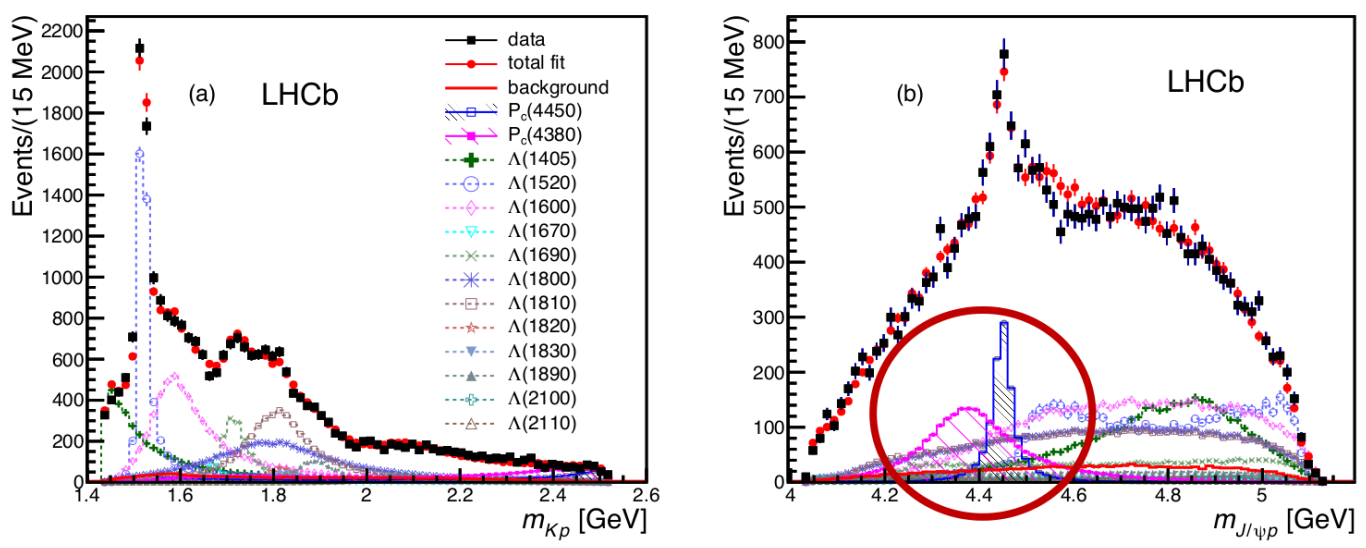

Figure 2. Dalitz plot projections of $\Lambda_{b}^{0} \rightarrow J / \psi p K^{-}$with superimposed the results from the amplitude analysis.

fit with resonance invariant mass, three helicity angles and two differences between decay planes has been applied to describe the data. It allowed interference between the decays $\Lambda_{b}^{0} \rightarrow J / \psi \Lambda^{*}$ and $\Lambda_{b}^{0} \rightarrow P_{c}^{+} K^{-}$, with $\Lambda^{*} \rightarrow p K^{-}$and $P_{c}^{+} \rightarrow J / \psi p$, where $P_{c}^{+}$indicates the pentaquark candidate. The amplitude model in the fit contains 14 well-defined $\Lambda^{*}$ states listed by Particle Data Group [6] and two $P_{c}^{+}$states: $P_{c}(4380)^{+}$and $P_{c}(4450)^{+}$. With a full amplitude analysis, the masses of these two states are measured to be $4380 \pm 8$ (stat) \pm 29 (syst) $\mathrm{MeV}$ and $4449.8 \pm 1.7$ (stat) \pm 2.5 (syst) $\mathrm{MeV}$, with the widths $205 \pm 18$ (stat) \pm 86 (syst) $\mathrm{MeV}$ and $39 \pm 5$ (stat) \pm 19 (syst) MeV, respectively. The preferred spin- 
parity values are $\left(3 / 2^{-}, 5 / 2^{+}\right),\left(3 / 2^{+}, 5 / 2^{-}\right)$and $\left(5 / 2^{+}, 3 / 2^{-}\right)$, where the first one is the $J^{P}$ assignment given by the best fit. This analysis has been extended with a model-independent approach [7] as first proposed by the BaBar collaboration for the $Z_{c}(4430)^{-}$analysis [8]. The model independent analysis gives similar results and allows to exclude that data can be described by the $p K^{-}$contributions alone. The evidence of the resonant character of the discovered pentaquark states was obtained observing the evolution of the complex amplitude in the Argand diagram for $P_{c}(4450)^{+}$, which is consistent with a rapid counter-clockwise change of the phase when its magnitude reaches the maximum, a behavior characteristic for a resonance.

\section{Exotic baryonic resonances in $\Lambda_{b}^{0} \rightarrow P_{c}^{+} \pi^{-}$}

A possible way for an independent confirmation of the discovered $P_{c}(4380)^{+}$and $P_{c}(4450)^{+}$states is to check whether such states also occur in the the Cabibbo-suppressed decay $\Lambda_{b}^{0} \rightarrow J / \psi p \pi^{-}$. Analyzing the same Run I dataset, but now in the Cabibbo suppressed mode, the signal yield was $1885 \pm 50$, about 15 times less than that of the Cabibbo-favored channel and with a relatively high background level [5]. The following possible contributions to the decay amplitude were taken into account in the analysis: $\Lambda_{b}^{0} \rightarrow J / \psi N^{*}$ with $N^{*} \rightarrow p \pi^{-}, \Lambda_{b}^{0} \rightarrow P_{c}^{+} \pi^{-}$with $P_{c}^{+} \rightarrow J / \psi p$, and $\Lambda_{b}^{0} \rightarrow Z_{c}(4200)^{-} p$ with $Z_{c}^{-} \rightarrow J / \psi \pi^{-}$where the $Z_{c}(4200)^{-}$has been reported by the Belle collaboration in the decay $B^{0} \rightarrow J / \psi K^{+} \pi^{-}$[9]. The $N^{*}$ resonances were modeled using relativistic Breit-Wigners, except the $N(1535)$, which is modeled by a Flatté lineshape, as it couples also to the near-threshold $n \eta$ mode. The full amplitude analysis used in the first pentaquark analysis has been applied to this Cabibbo suppressed $\Lambda_{b}^{0}$ decay. Both reduced and extended models of the $N^{*}$ contributions have been tested, the latter including a wider set of resonances and allowed couplings. Figure 3 shows the projections of the six-dimensional fit results to the background-subtracted data onto the $m\left(p \pi^{-}\right)$and $m(J / \psi p)$ mass distributions. The data were found to be consistent with the presence of the two exotic candidates, but the limited statistics did not allow to obtain high significances $(3.1 \sigma)$ for the two $P_{c}$ states, also due to the possible presence of the $Z_{c}$ exotic contribution.
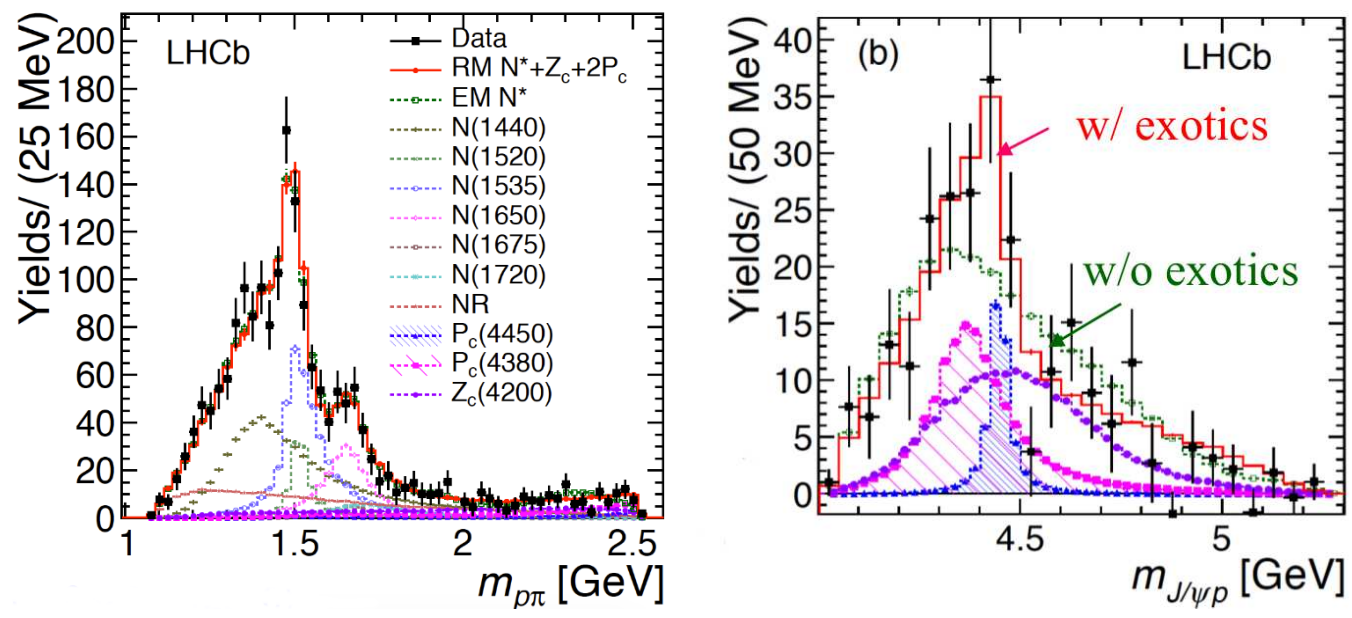

Figure 3. Dalitz plot projections of $\Lambda_{b}^{0} \rightarrow J / \psi p \pi^{-}$with superimposed the results from the amplitude analysis. 


\section{Confirmation of resonant nature of $Z_{c}(4430)^{-}$}

The $Z_{c}(4430)^{-}$state was first reported by the Belle collaboration [10] as a charged resonance structure in the $\psi^{\prime} \pi^{-}$invariant mass distribution in the decay $B^{0} \rightarrow \psi^{\prime} K^{+} \pi^{-}$. A relatively narrow peak has been found, $\Gamma \approx 45 \mathrm{MeV}$, in the $\psi^{\prime} \pi^{-}$mass spectrum with a mass of $4433 \pm 5 \mathrm{MeV}$. The BaBar collaboration could explain the enhancement as a reflection of the known $K^{*}$ states [8]. In 2013 Belle collaboration, using more data with about 2000 signal events, performed a full amplitude analysis of the same final state. They obtained a significance of $5.2 \sigma$ for the $Z_{c}(4430)^{-}$state, with a mass $M\left(Z_{c}\right)=4485 \pm 22 \pm 28$ $\mathrm{MeV}$ and width $\Gamma\left(Z_{c}\right)=200 \pm 41 \pm 26 \mathrm{MeV}$, favoring the $J^{P}=1^{+}$spin-parity assignment by more than $3.4 \sigma$.

The LHCb experiment collected about 25,000 $B^{0}$ signal events, a yield one order of magnitude larger than the BaBar or Belle datasets, and observed the $Z_{c}(4430)^{-}$with a significance larger than $13.9 \sigma$ [11]. The LHCb collaboration performed both a model independent analysis as well as a full amplitude analysis. The projection of the fit including the $Z_{c}(4430)^{-}$state is shown in Fig. 4 and evidences a good description of the data. The measured parameters are $M\left(Z_{c}\right)=4475 \pm 7_{-25}^{+15}$ $\mathrm{MeV}, \Gamma\left(Z_{c}\right)=172 \pm 13_{34}^{+37} \mathrm{MeV}$. The Argand diagram of the $Z_{c}(4430)^{-}$amplitude (Fig. 4) shows the resonance behavior for the first time. The spin-parity was measured to be $1^{+}$, by excluding other hypotheses by at least $9.7 \sigma$. For a charged charmonium state, $Z_{c}(4430)^{-}$has a minimum quark content of $c \bar{c} u \bar{d}$ which clearly does not fit into the traditional quark model.
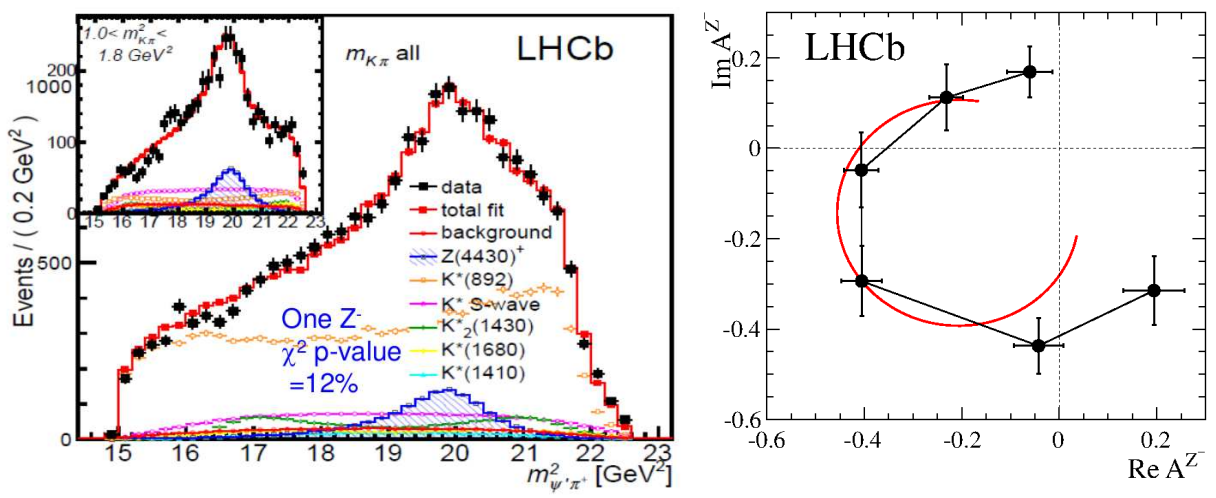

Figure 4. (Left) $\psi^{\prime} \pi^{-}$mass distribution from $\bar{B}^{0} \rightarrow \psi^{\prime} \pi^{-} K^{+}$with superimposed the results from the amplitude analysis. (Right) Argand diagram for $Z_{c}(4430)^{-}$.

\section{Study of $B \rightarrow J / \psi \phi K$}

There has been a great deal of experimental and theoretical interest in $J / \psi \phi$ mass structures in $B^{+} \rightarrow J / \psi \phi K^{+}$decays since the CDF collaboration presented $3.8 \sigma$ evidence for a near-threshold $X$ (4140) mass peak, with width $\Gamma=11.7 \mathrm{MeV}$ [12]. Much larger widths are expected for charmonium states at this mass, therefore it has been suggested that the $X(4140)$ peak could be a molecular state, a tetraquark state, a hybrid state or a rescattering effect. Subsequent measurements resulted in a confusing experimental situation. LHCb experiment has reconstructed $4289 \pm 151 \mathrm{~B}^{+} \rightarrow J / \psi \phi \mathrm{K}^{+}$ decays, with roughly uniform efficiency and a relatively low background across the entire $J / \psi \phi$ mass range [13]. A full amplitude analysis has been performed, including resonant contributions from 
Table 1. Parameters of the resonances decaying to $J / \psi \phi$ resulting from the amplitude analysis of $B^{+} \rightarrow J / \psi \phi K^{+}$

\begin{tabular}{lcccc}
\hline Resonance & $\sigma$ & $J^{P C}$ & $\mathrm{M}(\mathrm{MeV})$ & $\Gamma(\mathrm{MeV})$ \\
\hline $\mathrm{X}(4140)$ & 8.4 & $1^{++}$ & $4160 \pm 4_{-3}^{+5}$ & $83 \pm 21_{-14}^{+21}$ \\
$\mathrm{X}(4274)$ & 5.8 & $1^{++}$ & $4273 \pm 8_{-4}^{+17}$ & $56 \pm 11_{-11}^{+8}$ \\
$\mathrm{X}(4500)$ & 6.1 & $0^{++}$ & $4506 \pm 11_{-15}^{+12}$ & $92 \pm 21_{-20}^{+21}$ \\
$\mathrm{X}(4700)$ & 5.6 & $0^{++}$ & $4704 \pm 10_{-24}^{+14}$ & $120 \pm 31_{-33}^{+42}$ \\
\hline
\end{tabular}

$K^{*}$ resonances decaying to $\phi K^{+}$. Four resonant contributions in the $J / \psi \phi$ final state are found to contribute, whose parameters and quantum numbers are given in Table 1 . The fit results are superimposed to the $J / \psi \phi$ mass spectrum in fig. 5. It can be noted that the $X(4140)$ width is substantially larger than
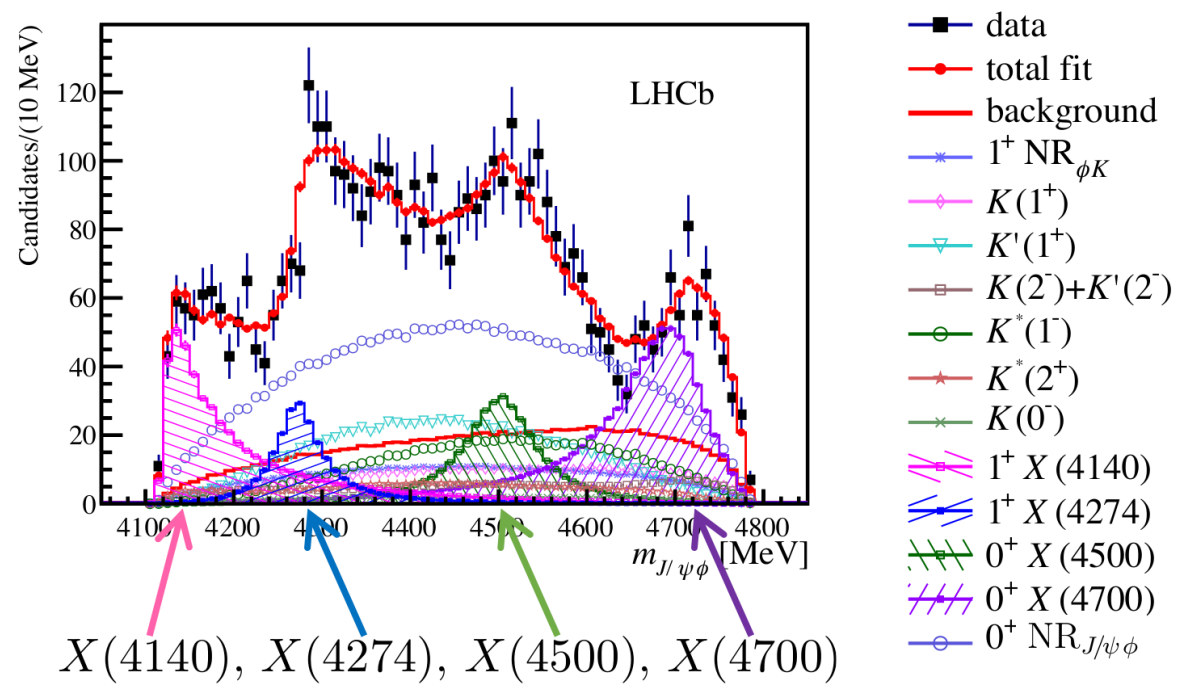

Figure 5. $J / \psi \phi$ mass projection with superimposed the fit result from the amplitude analysis of $B^{+} \rightarrow J / \psi \phi K^{+}$

previously determined. The interpretation of these states is still an open issue.

\section{Observation of five new $\Omega_{c}^{0}$ states}

The spectroscopy of singly charmed baryons $c q q^{\prime}$ is intricate. With three quarks and numerous degrees of freedom, many states are expected. At the same time, the large mass difference between the charm quark and the light quarks provides a natural way to understand the spectrum by using the symmetries provided by Heavy Quark Effective Theory (HQET). In recent years, considerable improvements have been made in the predictions of the properties of these heavy baryons. In many of these models, the heavy quark interacts with a $(q q)$ diquark, which is treated as a single object. These models predict seven states in the mass range 2.9-3.2 GeV. In particular, among the expected charmed baryon states the spectrum of $\Omega_{c}$ baryons, which have quark content css and isospin zero, is largely unknown: only the $\Omega_{c}$ and $\Omega_{c}(2770)^{0}$, presumed to be the $J^{P}=1 / 2^{+}$and $3 / 2^{+}$ground states, have been observed.

The $\mathrm{LHCb}$ experiment has reconstructed a large $\left(\approx 10^{6}\right.$ events) and high-purity $(83 \%)$ sample of $\Xi_{c}^{+}$baryons, reconstructed in the Cabibbo-suppressed decay mode $p K^{+} \pi^{-}$[14]. Surprisingly, in 
the $\Xi_{c}^{+} K^{-}$mass spectrum, shown in Fig. 6, five new, narrow excited $\Omega_{c}$ states are observed: the $\Omega_{c}(3000)^{0}, \Omega_{c}(3050)^{0}, \Omega_{c}(3066)^{0}, \Omega_{c}(3090)^{0}$, and $\Omega_{c}(3119)^{0}$. The measured masses and widths are reported in Table 2. The data indicate also the presence of a broad structure around $3188 \mathrm{MeV}$ that is fitted as a single resonance but could be produced in other ways, for example as a superposition of several states. In addition, the partially reconstructed decay $\Omega_{c}(3066)^{0}, \Omega_{c}(3090)^{0}$ and $\Omega_{c}(3119)^{0}$ in their possible decay to $\Xi_{c}^{\prime+} K^{-}$are observed via their feed-down in the low mass region. This observation has generated large interest, especially for the presence of very narrow states, and many phenomenological models have been proposed, including the possibility of the presence of pentaquark states.

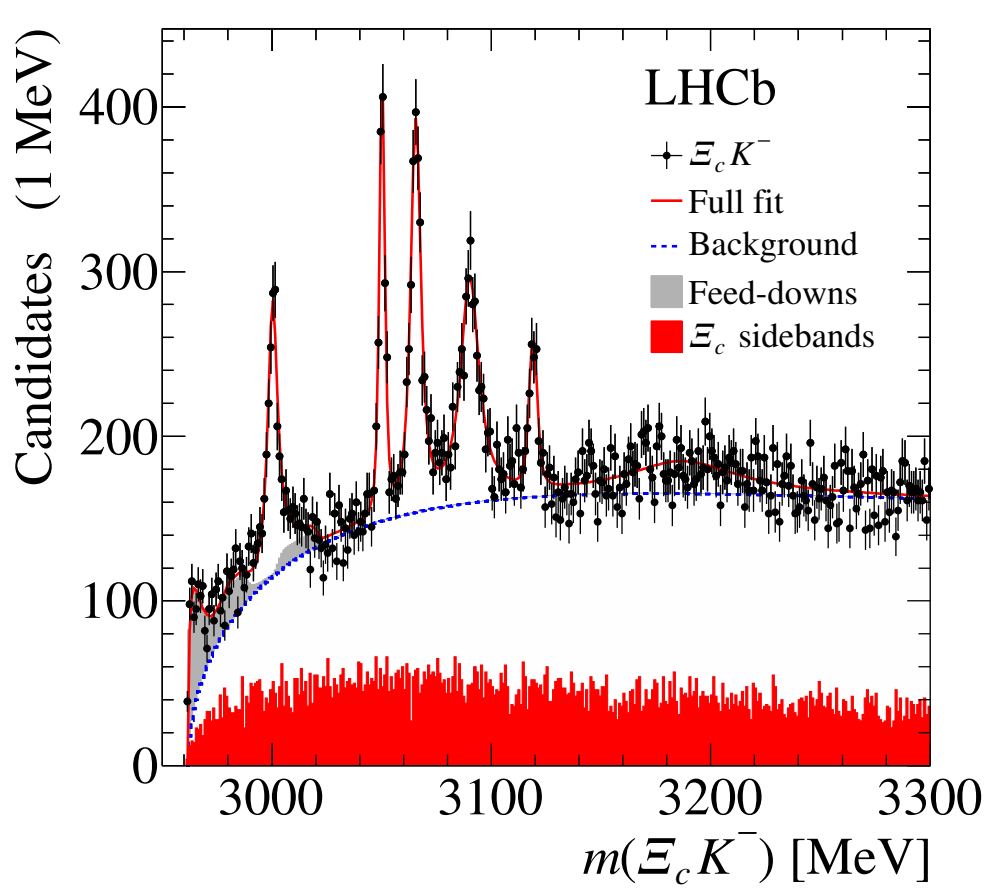

Figure 6. $\Xi_{c}^{+} K^{-}$mass spectrum with the observation of five new $\Omega_{c}$ states.

\section{Observation of the double charmed baryon $\Xi_{c c}^{++}$}

The constituent quark model of hadrons predicts the existence of three weakly decaying ground state $J^{P}=1 / 2^{+}$baryons with two charmed valence quarks: $\Xi_{c c}^{+}$(ccd) and $\Xi_{c c}^{++}$(ccu), which form an isospin doublet, and $\Omega_{c c}^{+}$(ccs). Several QCD computational formalisms have been used to predict their masses, lifetimes, relative production rates, and the spectroscopy of their excited states. The only previously published evidence of their existence comes from the observations of the $\Xi_{c c}^{+}$baryon claimed by the SELEX collaboration [15]. They observed narrow peaking structures at a mean mass of $3518.7 \pm 1.7 \mathrm{MeV}$ in the mass spectra of $\Lambda_{c}^{+} K^{-} \pi^{+}$(15.9 signal events over $6.1 \pm 0.5$ background events, $6.3 \sigma$ significance) and $p D^{+} K^{-}$(5.62 signal events over $1.38 \pm 0.13$ background events, $4.8 \sigma$ 
Table 2. Measured parameters of the five new $\Omega_{c}$ states.

\begin{tabular}{lcc}
\hline Resonance & Mass $(\mathrm{MeV})$ & $\Gamma(\mathrm{MeV})$ \\
\hline$\Omega_{c}(3000)^{0}$ & $3000.4 \pm 0.2 \pm 0.1_{-0.5}^{+0.3}$ & $4.5 \pm 0.6 \pm 0.3$ \\
$\Omega_{c}(3050)^{0}$ & $3050.2 \pm 0.1 \pm 0.1_{-0.5}^{+0.3}$ & $0.8 \pm 0.2 \pm 0.1$ \\
& & $<1.2 \mathrm{MeV}, 95 \% \mathrm{CL}$ \\
$\Omega_{c}(3066)^{0}$ & $3065.6 \pm 0.1 \pm 0.3_{-0.5}^{+0.3}$ & $3.5 \pm 0.4 \pm 0.2$ \\
$\Omega_{c}(3090)^{0}$ & $3090.2 \pm 0.3 \pm 0.5_{-0.5}^{+0.3}$ & $8.7 \pm 1.0 \pm 0.8$ \\
$\Omega_{c}(3119)^{0}$ & $3119.1 \pm 0.3 \pm 0.9_{-0.5}^{+0.3}$ & $1.1 \pm 0.8 \pm 0.4$ \\
& & $<2.6 \mathrm{MeV}, 95 \% \mathrm{CL}$ \\
\hline$\Omega_{c}(3188)^{0}$ & $3188.1 \pm 4.8 \pm 12.7$ & $60 \pm 15 \pm 11$ \\
\hline
\end{tabular}

significance). The lifetime of the state was unexpectedly short (less than $33 \mathrm{fs}$ at the $90 \%$ confidence level) and had a production rate several orders of magnitude larger than theoretical predictions for their hyperon-beam on fixed target collisions. Identification of the state as the $\Xi_{c c}^{+}$baryon implied that $20 \%$ of all $\Lambda_{c}^{+}$baryons observed by the SELEX experiment were produced in decays of $\Xi_{c c}^{+}$baryons.

LHCb experiment has searched for double charmed baryon states using the decay mode $\Xi_{c c}^{++} \rightarrow$ $\Lambda_{c} K^{-} \pi^{+} \pi^{+}$, with $\Lambda_{c} \rightarrow p K^{-} \pi^{+}$(which has a $10 \%$ branching fraction) [16]. The $\Lambda_{c} K^{-} \pi^{+} \pi^{+}$mass spectrum is shown in Fig. 7 where a prominent signal can be observed.

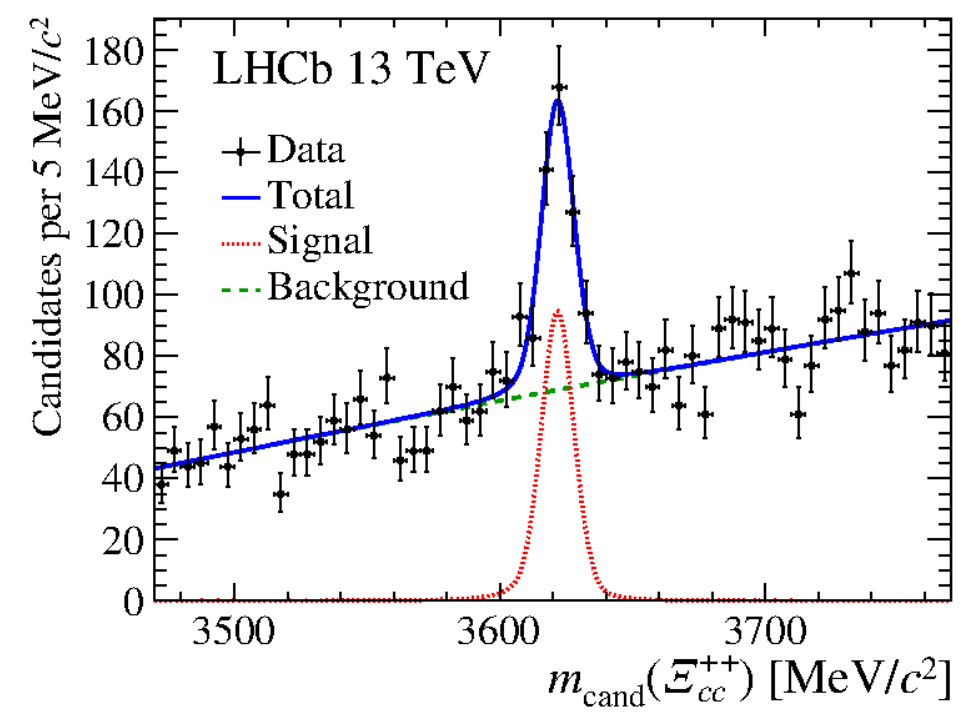

Figure 7. $\Lambda_{c} K^{-} \pi^{+} \pi^{+}$mass spectrum with the observation of $\Xi_{c c}^{++}$.

LHCb observes $313 \pm 33$ decays with a significance of $>12 \sigma$ of a $\Xi_{c c}^{++}$state that has a mass of $m\left(\Xi_{c c}^{++}\right)=3621.40 \pm 0.72$ (stat) \pm 0.27 (syst) $\pm 0.14\left(\Lambda_{c}\right) \mathrm{MeV}$ and a width that is much smaller than experimental resolution and that is consistent with a particle that flies a significant distance before decaying. 
This is the first published observation of the $\Xi_{c c}^{++}$baryon. The published SELEX $\Xi_{c c}^{+}$state has a mass that is $103 \pm 2 \mathrm{MeV}$ smaller than the $\Xi_{c c}^{++}$state, so the two observations are inconsistent with being isospin partners. A consistent interpretation of the two observations may prove challenging.

\section{Charmed baryon spectroscopy using $\Lambda_{b}$ decays}

LHCb experiment has performed a full amplitude analysis of the Cabibbo-favoured decay $\Lambda_{b} \rightarrow$ $D^{0} p \pi^{-}$[17]. This $\Lambda_{b}$ decay mode allows to study baryon spectroscopy in the $D^{0} p$ final state and the determination of the quantum numbers of the observed states. The analysis was possible due to the large statistics and the clean $\Lambda_{b}$ reconstructed signal (11,200 events, $86 \%$ purity).

Figure 8 shows the fit projection on the $D^{0} p$ mass spectrum, where three $\Lambda_{c}^{+}$states are observed, with parameters given in Table 3 .

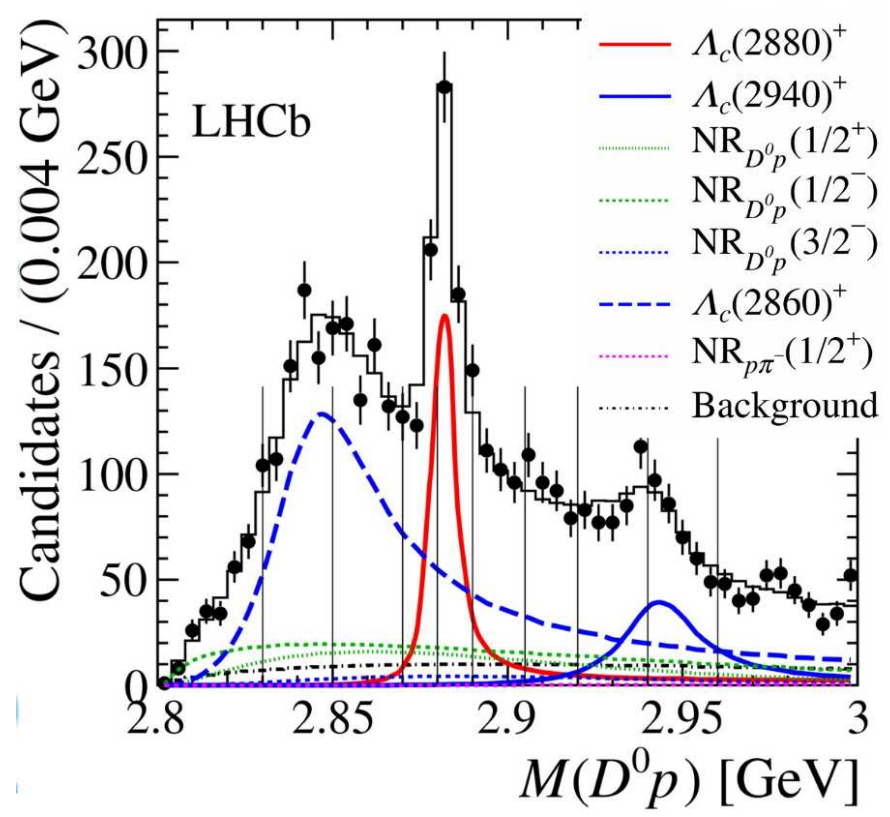

Figure 8. $D^{0} p$ mass projection with the results from the amplitude analysis of $\Lambda_{b} \rightarrow D^{0} p \pi^{-}$

The two $\Lambda_{c}(2880)^{+}$and $\Lambda_{c}(2940)^{+}$states have been previously observed by BaBar [18] experiment, but without a spin-parity determination. For $\Lambda_{c}(2860)^{+}$the present analysis represents a first observation.

Table 3. Parameters of resonances decaying to $D^{0} p$ from the amplitude analysis of $\Lambda_{b} \rightarrow D^{0} p \pi^{-}$. The tabulated errors are statistic, systematic and related to the fitting model.

\begin{tabular}{cccc}
\hline Resonance & $J^{P C}$ & $\mathrm{M}(\mathrm{MeV})$ & $\Gamma(\mathrm{MeV})$ \\
\hline$\Lambda_{c}(2860)^{+}$ & $3 / 2^{+}$ & $2856.1_{-1.7}^{+2.0} \pm 0.5_{-5.6}^{+1.1}$ & $67.6_{8.1}^{+10.1} \pm 1.4_{-20.0}^{+5.9}$ \\
$\Lambda_{c}(2880)^{+}$ & $5 / 2^{+}$ & $2881.75 \pm 0.29 \pm 0.07_{-0.20}^{+0.14}$ & $5.43_{0.71}^{+0.77} \pm 0.29_{-0.00}^{+0.75}$ \\
$\Lambda_{c}(2940)^{+}$ & $3 / 2^{-}$ & $2944.8_{-2.5}^{+3.5} \pm 0.4_{-4.6}^{+0.1}$ & $27.7_{-6.0}^{+8.2} \pm 0.9_{-10.4}^{+5.2}$ \\
\hline
\end{tabular}




\section{Conclusions}

$\mathrm{LHCb}$ is a flavor factory, exploring a large set of physics topics. In particular, in the spectroscopy field, many new unexplored regions are being studied. These analyses are producing unexpected results, such as the discovery of "exotic" states, or the observation of many unexpected resonances and particles. Basic ingredients of these results are: the high statistics and purity of the final states and highly sophisticated and newly developed full amplitude analyses. This field is in rapid development and much more experimental and theoretical work is needed to understand the full pattern. Many more analyses are underway, making use of the large amount of data which are being collected at LHC.

\section{References}

[1] M. Gell-Mann, Phys. Lett. 8, 214 (1964).

[2] A. Dzierba, C. Mayer and A. Szczepaniak, hep-ex/04120 (2005).

[3] B. Aubert et al. [BaBar Collaboration], Phys. Rev. Lett. 95, 042002 (2005). J. M. Link et al. [FOCUS Collaboration], Phys. Lett. B 639, 604 (2006).

[4] R. Aaij et al. [LHCb Collaboration], Phys. Rev. Lett. 115, 072001 (2015).

[5] R. Aaij et al. [LHCb Collaboration], Phys. Rev. Lett. 117, no. 8, 082003 (2016).

[6] C. Patrignani et al. (Particle Data Group), Chin. Phys. C, 40, 100001 (2016) and 2017 update.

[7] R. Aaij et al. [LHCb Collaboration], Phys. Rev. Lett. 117, no. 8, 082002 (2016).

[8] B. Aubert et al. [BaBar Collaboration], Phys. Rev. D 79, 112001 (2009).

[9] K. Chilikin et al. [Belle Collaboration], Phys. Rev. D 90, no. 11, 112009 (2014).

[10] S. K. Choi et al. [Belle Collaboration], Phys. Rev. Lett. 100, 142001 (2008).

[11] R. Aaij et al. [LHCb Collaboration], Phys. Rev. Lett. 112, no. 22, 222002 (2014).

[12] CDF collaboration, T. Aaltonen et al., [CDF Collaboration], Phys. Rev. Lett. 102, 242002 (2009).

[13] R. Aaij et al. [LHCb Collaboration], Phys. Rev. Lett. 118, 022003 (2017).

[14] R. Aaij et al. [LHCb Collaboration], Phys. Rev. Lett. 118, 182001 (2017).

[15] A. Ocherashvili et al. [SELEX Collaboration], Phys. Lett. B 628, 18 (2005).

[16] R. Aaij et al. [LHCb Collaboration], Phys. Rev. Lett. 119, 112001 (2017).

[17] R. Aaij et al. [LHCb Collaboration], JHEP 1705, 030 (2017).

[18] B. Aubert et al. [BaBar Collaboration], Phys. Rev. Lett. 98, 012001 (2007). 